\title{
Value of radial probe endobronchial ultrasound-guided localization of solitary pulmonary nodules with the combination of ultrathin bronchoscopy and methylene blue prior to video-assisted thoracoscopic surgery
}

\author{
CHUN-HUA XU ${ }^{1,2}$, LI-KE YU ${ }^{1,2}$, LAN CAO $^{2}$, RUSONG YANG $^{3}$, \\ JUN YAN $^{4}$, ZHENG-CHENG LIU ${ }^{3}$ and YAN WANG ${ }^{5}$
}

\author{
${ }^{1}$ Department of Respiratory Medicine, Nanjing Chest Hospital; ${ }^{2}$ Nanjing Clinical Center of Respiratory \\ Diseases and Imaging, Nanjing, Jiangsu 210029; ${ }^{3}$ Department of Thoracic Surgery, Nanjing Chest Hospital, \\ Nanjing, Jiangsu 210029; ${ }^{4}$ MOE Key Laboratory, Model Animal Research Center, Nanjing University, Nanjing, \\ Jiangsu 210061; ${ }^{5}$ Department of Radiology, Nanjing Chest Hospital, Nanjing, Jiangsu 210029, P.R. China
}

Received February 24, 2015; Accepted May 15, 2015

DOI: $10.3892 / \mathrm{mco} .2016 .913$

\begin{abstract}
The aim of this study was to assess the clinical value of radial probe endobronchial ultrasound (RP-EBUS)-guided localization of solitary pulmonary nodules (SPNs) with the combination of ultrathin bronchoscopy and methylene blue prior to video-assisted thoracoscopic surgery (VATS). An ultrathin bronchoscope was used to localize the lesions under RP-EBUS guidance in 48 patients (18 men and 30 women; age range, $41-72$ years; mean age, 54 years), who subsequently underwent VATS resection. The lesion size, distance from the parietal pleura, localization time and complications were evaluated. The RP-EBUS-guided localization success rate was $72.9 \%$. The lesion size \pm standard deviation was $12.8 \pm 4.2 \mathrm{~mm}$ and the mean distance from the parietal pleura was $11.2 \pm 9.7 \mathrm{~mm}$. The mean localization time was $15.7 \pm 8.3 \mathrm{~min}$. The major complication of RP-EBUS-guided localization was asymptomatic hemorrhage in 4 patients $(8.3 \%)$. The VATS resection success rate was $95.8 \%$. In terms of pathological type, the 48 lesions included atypical adenomatous hyperplasia $(\mathrm{n}=4)$, adenocarcinoma in situ $(\mathrm{n}=5)$, minimally invasive adenocarcinoma $(\mathrm{n}=7)$, adenocarcinoma $(\mathrm{n}=18)$, squamous cell carcinoma $(n=1)$, inflammation $(n=6)$, hamartoma $(n=4)$ and tuberculosis $(n=3)$. Therefore, RP-EBUS-guided localization with the combination of an ultrathin bronchoscope and methy-
\end{abstract}

Correspondence to: Professor Li-Ke Yu or Professor Rusong Yang, Department of Respiratory Medicine, Nanjing Chest Hospital, 215 Guangzhou Road, Nanjing, Jiangsu 210029, P.R. China

E-mail: yulike_doctor@126.com

E-mail: yangrusong_nj@126.com

Key words: solitary pulmonary nodule, localization, video-assisted thoracoscopic surgery, radial probe endobronchial ultrasound-guided bronchoscopy lene blue prior to VATS resection is a promising technique for SPNs, it plays an important role in the accurate localization of SPNs and it is an effective and safe technique to assist VATS resection of such nodules.

\section{Introduction}

The solitary pulmonary nodule (SPN) is defined as an isolated, single lesion of round or oval shape, with a diameter of $\leq 3 \mathrm{~cm}$, which is located within the lung parenchyma, surrounded entirely by gas-containing lung tissue. Such lesions are not accompanied by lung atelectasis, hilar enlargement or pleural effusion $(1,2)$. With the established role of computed tomography (CT) screening for lung cancer and the wide application of high-resolution CT, SPNs are detected at an increasing rate (3-5). SPN management basically comprises the implementation of immediate surgical treatment $(6,7)$. Over the last decade, video-assisted thoracoscopic surgery (VATS) has become a useful tool in the diagnosis and treatment of SPNs $(8,9)$. However, due to the small size of the nodules, preoperative localization is crucial for the success of VATS.

The preoperative localization methods include microvascular embolisation coils, hookwire insertion and injection of dye (10-15). Each method has its merits and drawbacks. We developed a new technique using a combination of methylene blue and an ultrathin bronchoscope under radial probe endobronchial ultrasound (RP-EBUS) guidance. This technique was applied in 48 patients and achieved effective, safe and convenient SPN localization.

\section{Materials and methods}

Patients. Between January, 2013 and September, 2014, RP-EBUS-guided SPN localization with an ultrathin bronchoscope and methylene blue, followed by VATS, was conducted on 48 SPNs from 48 patients who underwent CT examination at the Nanjing Clinical Center of Respiratory Diseases and 
Imaging (Nanjing, China). The study included 18 men and 30 women, with a mean age of 54 years (range, $41-72$ years). Of the 48 patients, 12 had a cancer history. The patient characteristics are summarized in Table I. The study protocol was approved by the Ethics Committee of the Nanjing Chest Hospital and all the patients provided written informed consent.

$R P$-EBUS-guided combination of ultrafine bronchoscopy and methylene blue localization. Each patient had been diagnosed with a pulmonary nodule on a CT scan of the chest, measuring $10 \mathrm{~mm}$ (Fig. 1). RP-EBUS scanning was conducted under anesthesia, with percutaneous blood oxygen saturation monitoring and continuous electrocardiography during the examination process. A 2.0-mm, 20-MHz radial mechanical transducer type endobronchial ultrasonic probe (UM-BS20-26R; Olympus, Tokyo, Japan) with a flexible balloon sheath (MAJ-643R; Olympus) was introduced through the $2.8-\mathrm{mm}$ channel of a flexible bronchoscope (XBF-22L; Olympus). The probe was connected to the endobronchial ultrasonography unit (EU-M 20/30; Olympus). The visible bronchial segment was examined until the characteristic ultrasound signal indicating the presence of a solid lesion (Fig. 2). The EBUS probe was removed and $0.3 \mathrm{ml}$ methylene blue was injected at the marked point, according to the angle and depth data.

VATS. Thoracoscopic segmentectomy was performed under general anesthesia using single-lung ventilation. The technique utilized three incisions. The observation port was $\sim 1 \mathrm{~cm}$ in length at the midaxillary line in the 6 th or 7 th intercostal space. The main operation port was placed in the 3rd or 4th intercostal space at the anterior axillary line. The accessory operation port was usually placed at the posterior axillary line in the 6th intercostal space. Both operation ports were $\sim 2 \mathrm{~cm}$ in length.

The methylene blue was visualized during the exploration and the location of the lesion was confirmed (Fig. 3). The initial step was dissection of the hilar lymph nodes to ensure safe sublobar resection. The lymph nodes were sent for frozen section analysis and sublobar resection was abandoned if positive nodes were identified.

The approach to thoracoscopic segmentectomy begun with ligation of the segmental pulmonary vein and artery using thoracoscopic linear mechanical staplers. The bronchus was further isolated and dissected by stapler. Subsequently, the ipsilateral lung was temporarily reinflated to help identify the segmental fissures. After the fissures were marked with electrocautery and squeezed by long Kelly clamps, the segmental plane was finished with an endostapler. In certain cases, we had to convert to a thoracotomy due to problems such as adhesions or failure of localization.

\section{Results}

Characteristics of the SPNs. In 35 (72.9\%) of the 48 patients, the methylene blue was visualized in or adjacent to the pulmonary nodule as documented following VATS resection (Fig. 4). The maximal diameter of the SPNs, their distance from the pleural surface and their histological diagnosis are summarized in Tables II and III. The diameter of the nodules \pm standard deviation (SD) was $12.8 \pm 4.2 \mathrm{~mm}$ and located at a distance of
Table I. Patient characteristics $(n=48)$.

Characteristics

No. of patients $(\%)$

$\begin{array}{ll}\text { Age, years } & \\ \geq 60 & 20(41.7) \\ <60 & 28(58.3)\end{array}$

Gender

Male

Female

Cancer history

Yes

No

Smoking history

Smoker

Non-smoker

$33(68.7)$

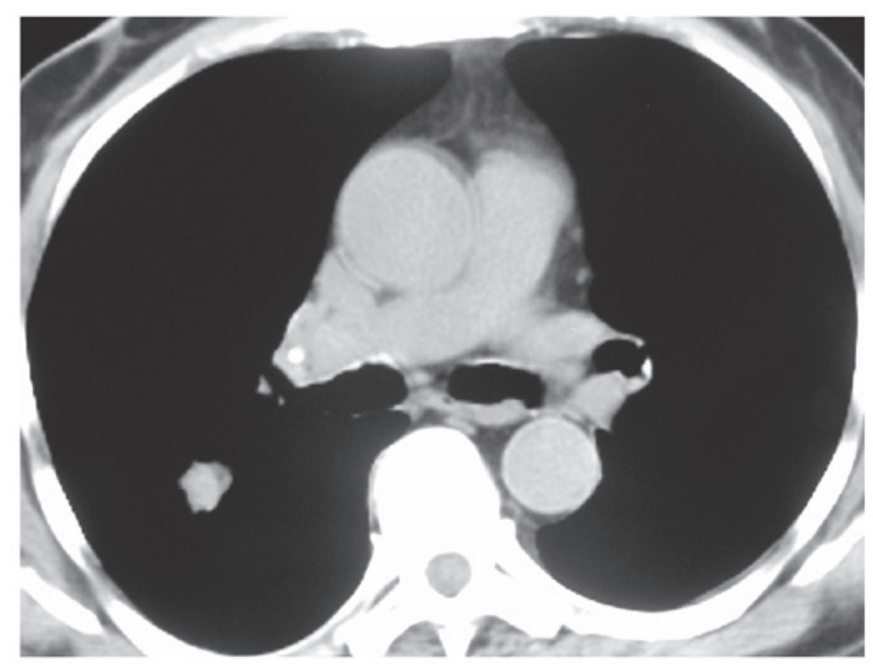

Figure 1. Chest CT scan revealing a nodule in the lateral part of the lower lobe of the right lung, measuring $12 \times 10 \mathrm{~mm}$.

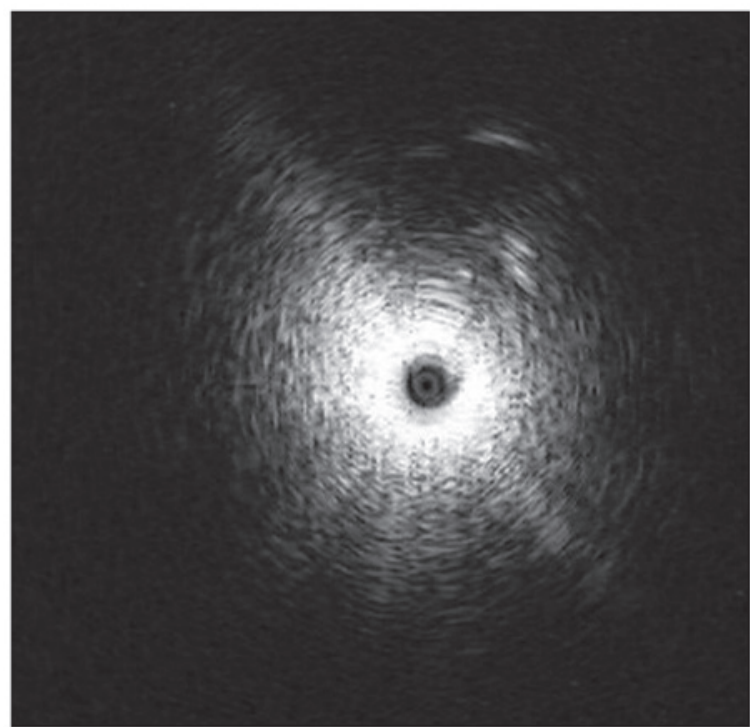

Figure 2. Radial probe endobronchial ultrasound image of a solitary pulmonary nodule with a measured diameter of $12 \mathrm{~mm}$. 
Table II. Characteristics of the SPNs $(n=48)$.

\begin{tabular}{lc}
\hline Characteristics & Value \\
\hline Diameter, mm & $12.8 \pm 4.2$ \\
Distance from pleural surface, mm & $11.2 \pm 9.7$ \\
Location, no. (\%) & $12(25.0)$ \\
Upper lobe of right lung & $10(20.8)$ \\
Lower lobe of right lung & $16(33.3)$ \\
Upper lobe of left lung & $10(20.8)$ \\
Lower lobe of left lung & $30(62.5)$ \\
Density, no. (\%) & \\
GGN & \\
\hline SPN, solitary pulmonary nodule; GGN, ground-glass nodule. \\
\hline
\end{tabular}

Figure 3. Intraoperative thoracoscopic view of a wedge resection of a pulmonary nodule that was preoperatively marked by methylene blue.

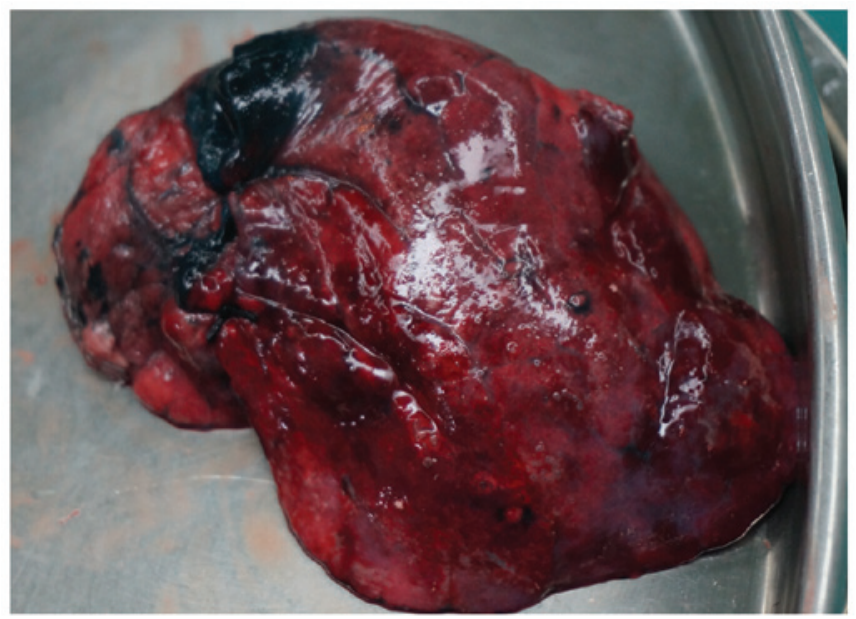

Figure 4. Lesion raised and resected under video-assisted thoracic surgery.

11.2 $\pm 9.7 \mathrm{~mm}$ from the pleural surface. A total of 35 nodules were malignant and 13 were benign. A total of 12 lesions were located in the right upper lobe, 10 were in the right lower lobe, 16 in the left upper lobe and 10 in the left lower lobe.
Table III. Histological diagnosis of SPNs.

\begin{tabular}{lc}
\hline Types & No. of patients $(\%)$ \\
\hline Benign & $13(27.1)$ \\
Hamartoma & $4(8.3)$ \\
Pulmonary tuberculosis & $3(6.3)$ \\
Infammatory pseudotumor & $6(12.5)$ \\
Malignant & $35(72.9)$ \\
Atypical adenomatous hyperplasia & $4(8.3)$ \\
Adenocarcinoma in situ & $5(10.4)$ \\
Minimally invasive adenocarcinoma & $7(14.6)$ \\
Adenocarcinoma & $18(37.5)$ \\
Squamous cell carcinoma & $1(2.1)$ \\
\hline
\end{tabular}

SPN, solitary pulmonary nodule.

Table IV. Characteristics of RP-EBUS-guided SPN localization with a combination of an ultrafine bronchoscope and methylene blue.

\begin{tabular}{lc}
\hline Characteristics & Values \\
\hline Complications, no. (\%) & \\
Pneumothorax & $0(0.0)$ \\
Hemorrhage & $4(8.3)$ \\
Pneumothorax and hemorrhage & $0(0.0)$ \\
Duration, min & \\
Range & $10-28$ \\
Mean & 15 \\
Ultrafine bronchoscope insertions, no. & \\
Range & $3-6$ \\
Mean & 3
\end{tabular}

RP-EBUS, radial probe endobronchial ultrasound; SPN, solitary pulmonary nodule.

$R P$-EBUS-guided SPN localization. The duration of localization, measured from the administration of local anesthesia, ranged between 10 and $28 \mathrm{~min}$ (mean, $15 \mathrm{~min}$ ) and the number of ultrafine bronchoscope insertions or adjustments ranged between 3 and 6 (mean, 3). The only complication of RP-EBUS-guided localization with the combination of an ultrafine bronchoscope and methylene blue was asymptomatic hemorrhage, which was observed in $8.3 \%$ of the patients. VATS was performed in all 48 patients; 38 cases underwent wedge resection, including 13 benign and 25 malignant lesions and the remaining 8 patients underwent lobectomy and lymphadenectomy. The mean surgery duration \pm SD for wedge resection and lobectomy was $25 \pm 8$ and $90 \pm 30 \mathrm{~min}$, respectively.

In two cases the procedure was converted to thoracotomy: One SPN strongly adhered to the pleural surface and the other was difficult to localize. The characteristics of the RP-EBUS-guided localization with an ultrafine bronchoscope and methylene blue and subsequent VATS are summarized in Table IV. 


\section{Discussion}

VATS is a useful minimally invasive procedure for the diagnosis and treatment of peripheral small pulmonary nodules. However, as such lesions may not be visible or palpable during VATS, a conversion from VATS to thoracotomy is occasionally conducted following failure to localize these lesions $(16,17)$. Therefore, it is crucial to accurately localize lesions prior to VATS, particularly in the case of small or faint nodules.

The most common localization method is the CT-guided insertion of a hookwire. However, this technique is associated with the development of pneumothorax and wires may be dislodged with movement (18). The most commonly used dye, methylene blue, may diffuse quickly to the uninvolved pleural surface and make localization difficult (19).

In the present study, 35 lesions were successfully localized. The mean duration of localization $\pm \mathrm{SD}$ was $15.7 \pm 8.3 \mathrm{~min}$ and the mean surgery duration for wedge resection and lobectomy was $25 \pm 8$ and $90 \pm 30$ min, respectively. All 48 lesions were pathologically examined. Malignant lesions accounted for $72.9 \%$ of the cases; this result was similar to previous reports (20-22). We suggest that this may be due to the benign diagnoses of certain patients in this study, who were selected based on imaging results rather than pathological diagnosis.

Although the RP-EBUS-guided method appears to be inferior to the CT-guided hookwire system in terms of successful localization, our technique provides several distinct advantages over other reported techniques, such as introduction of the probe through a natural lumen, absence of pneumothorax, low bleeding rate, no air embolism and no radiation damage.

Our study had several limitations, including the small number of patients and the fact that our localization method was not compared to other methods. Further comparative studies are required to provide a definitive answer on the optimal localization method prior to VATS.

In conclusion, RP-EBUS-guided combination of ultrafine bronchoscopy and methylene blue for the localization of SPNs prior to VATS is an effective, safe and convenient localization method.

\section{Acknowledgements}

The present study was supported in part by a grant from 'Twelve-Five Plan', the major program of Nanjing Medical Science and Technique Development Foundation (Molecular Mechanism Study on Metastasis and Clinical Efficacy Prediction of Non-small Cell Lung Cancer) to Li-Ke Yu.

\section{References}

1. Ost D, Fein AM and Feinsilver SH: Clinical practice. The solitary pulmonary nodule. N Engl J Med 348: 2535-2542, 2003.

2. Xu C, Hao K, Song Y, et al: Early diagnosis of solitary pulmonary nodules. J Thorac Dis 5: 830-840, 2013
3. Aberle DR, DeMello S, Berg CD, et al; National Lung Screening Trial Research Team: Results of the two incidence screenings in the National Lung Screening Trial. N Engl J Med 369: 920-931, 2013.

4. McWilliams A, Tammemagi MC, Mayo JR, et al: Probability of cancer in pulmonary nodules detected on first screening CT. N Engl J Med 369: 910-919, 2013.

5. Patel VK, Naik SK, Naidich DP, et al: A practical algorithmic approach to the diagnosis and management of solitary pulmonary nodules: part 1: radiologic characteristics and imaging modalities. Chest 143: 825-839, 2013.

6. Cummings SR, Lillington GA and Richard RJ: Estimating the probability of malignancy in solitary pulmonary nodules. A Bayesian approach. Am Rev Respir Dis 134: 449-452, 1986.

7. Mery CM, Pappas AN, Bueno R, et al: Relationship between a history of antecedent cancer and the probability of malignancy for a solitary pulmonary nodule. Chest 125: 2175-2181, 2004.

8. Liao H, Pu Q, Mei J, et al: Value of video-assisted thoracic surgery core needle biopsy in the selection of surgical approaches for indeterminate pulmonary nodules. Ann Thorac Surg 95: 772, 2013.

9. Bernard A: Resection of pulmonary nodules using video-assisted thoracic surgery. The Thorax Group. Ann Thorac Surg 61: 202-204, 1996.

10. Powell TI, Jangra D, Clifton JC, et al: Peripheral lung nodules: fluoroscopically guided video-assisted thoracoscopic resection after computed tomography-guided localization using platinum microcoils. Ann Surg 240: 481-488, 2004.

11. Saito H, Minamiya Y, Matsuzaki I, et al: Indication for preoperative localization of small peripheral pulmonary nodules in thoracoscopic surgery. J Thorac Cardiovasc Surg 124: 1198-1202, 2002.

12. Chen W, Chen L, Yang S, et al: A novel technique for localization of small pulmonary nodules. Chest 131: 1526-1531, 2007.

13. Kastl S, Langwieler TE, Krupski-Berdien G, et al: Percutaneous localization of pulmonary nodules prior to thoracoscopic surgery by CT-guided hook-wire. Anticancer Res 26: 3123-3126, 2006.

14. Partik BL, Leung AN, Müller MR, et al: Using a dedicated lung-marker system for localization of pulmonary nodules before thoracoscopic surgery. AJR Am J Roentgenol 180: 805-809, 2003.

15. Chella A, Lucchi M, Ambrogi MC, et al: A pilot study of the role of TC-99 radionuclide in localization of pulmonary nodular lesions for thoracoscopic resection. Eur J Cardiothorac Surg 18: 17-21, 2000.

16. Chen S, Zhou J, Zhang J, et al: Video-assisted thoracoscopic solitary pulmonary nodule resection after CT-guided hookwire localization: 43 cases report and literature review. Surg Endosc 25: 1723-1729, 2011.

17. Suzuki K, Nagai K, Yoshida J, et al: Video-assisted thoracoscopic surgery for small indeterminate pulmonary nodules: indications for preoperative marking. Chest 115: 563-568, 1999.

18. Horan TA, Pinheiro PM, Araújo LM, et al: Massive gas embolism during pulmonary nodule hook wire localization. Ann Thorac Surg 73: 1647-1649, 2002.

19. Iwasaki Y, Nagata K, Yuba T, et al: Fluoroscopy-guided barium marking for localizing small pulmonary lesions before video-assisted thoracic surgery. Respir Med 99: 285-289, 2005.

20. Pittet O, Christodoulou M, Pezzetta E, et al: Video-assisted thoracoscopic resection of a small pulmonary nodule after computed tomography-guided localization with a hook-wire system. Experience in 45 consecutive patients. World J Surg 31: 575-578, 2007.

21. Ciriaco P, Negri G, Puglisi A, et al: Video-assisted thoracoscopic surgery for pulmonary nodules: rationale for preoperative computed tomography-guided hookwire localization. Eur J Cardiothorac Surg 25: 429-433, 2004.

22. Hirai S, Hamanaka Y, Mitsui N, et al: Role of video-assisted thoracic surgery for the diagnosis of indeterminate pulmonary nodule. Ann Thorac Cardiovasc Surg 12: 388-392, 2006. 